\section{SMGrøup}

\section{SM}

\section{Otolaryngology}

Article Information

Received date: Dec 18, 2017

Accepted date: Dec 20, 2017

Published date: Mar 21, 2018

*Corresponding author

Muhammet Recai Mazlumoglu, Otorhinolaryngology Clinic, Hinis Sehit Yavuz Yurekseven State Hospital, 25100, Erzurum, Turkey, Tel: +90 542435 5835; Fax: +90 235 1015;

Email:dr.mazlumoglu@gmail.com

Distributed under Creative Commons CC-BY 4.0

Keywords Robot; Surgery; Total Laryngectomy; Cancer

Article DOI 10.36876/smotol.1014
Review Article

\section{Transoral Robotic Total Laryngectomy for Laryngeal Cancer and Other Diseases of the Larynx}

\author{
Muhammet Recai Mazlumoglu* \\ Otorhinolaryngology Clinic, Hinis Sehit Yavuz Yurekseven State Hospital, Turkey
}

\section{Abstract}

Total laryngectomy can be performed for the treatment of laryngeal diseases including laryngeal cancer, chronic aspiration, and bilateral vocal cord paralysis, glottic and subglottic stenosis. In case of chronic aspiration, glottic and subglottic stenosis total laryngectomy can be the last chance in extremely rare situations. Because of the high morbidity associated with this procedure, the development of a new treatment approach is paramount. Transoral robotic surgery is the newest treatment alternative. Here, we review published work on this approach.

\section{Introduction}

Head and neck squamous cell carcinoma is the sixth most common cancer. Each year around 500,000 new cases are diagnosed worldwide [1]. Laryngeal cancer is one of the most common forms of head and neck squamous cell carcinoma.

The introduction of robotic surgical systems has led to rapid developments in the treatment of head and neck cancer. While the focus was initially on oropharyngeal tumors, the development of new applications has allowed the use of robotic surgical techniques in anatomical regions such as the larynx. The procedure was first used to perform supraglottic laryngectomy and then total laryngectomy $[2,3]$.

Transoral Robotic Surgery (TORS) was developed in 2005, and is now widely accepted as a convenient and reliable technique. Subsequently, several case series [4-6] and cadaver studies $[7,8]$ related to Transoral Robotic Total Laryngectomy (TORTL) have been published.

\section{Preoperative Evaluation}

As TORTL is a new procedure, patient selection is important to avoid unexpected complications. However, because few studies have investigated this approach, no consensus has been reached on guidelines for patient selection.

For procedures such as thyroidectomy, parathyroidectomy, and neck dissection, robotic surgery is contraindicated for overweight patients, patients undergoing neck radiotherapy, and patients who have undergone previous neck surgery [9]. However, the findings of previous studies suggest that these factors are not contraindications for TORTL. Smith et al. [6] reported that in a case series of seven patients, six had undergone chemotherapy and/or radiotherapy, and TORTL was used as salvage therapy in those patients. Furthermore, TORTL has been used to treat patients with subglottic chondrosarcoma, laryngeal chondronecrosis, bilateral vocal cord paralysis, subglottic stenosis due to prolonged intubation, chronic aspiration due to neurological disease, and subglottic adenoid cystic carcinoma [4-6]. Previous investigators have preoperatively assessed the oropharynx, hypopharynx, and larynx using TORTL preoperative planning to assess anatomical suitability for the procedure [4-6]. Smith et al. [6] did not use TORTL in patients who had an anteriorly located larynx and a narrow mandibular arch. Furthermore, Schuler et al. [8] reported that TORTL was not suitable for patients with prelaryngeal fat tissue or postcricoid invasion. In addition, the same authors reported that neck dissection should not be performed simultaneously.

\section{Surgical Procedure}

In addition to the robotic system, special retractors, such as the LARS4 (Fentex Medical, Neuhausen, Germany) or the WO-FK (Gyrus ACMI, Tuttlingen, Germany) are required to widen the surgical area [3].

The patient is placed in the supine position with the head in the extensor position. The bed is positioned so the foot of the bed is toward the anesthesia equipment [4].

\title{
ISSN: 2574-2418
}


The procedure begins with a standard tracheostomy skin incision. A tracheostomy is performed by making a $4 \mathrm{~cm}$ long incision between the sternal notch and the cricoid cartilage. The superior subplatysmal flap is raised, and the strap muscles are divided to expose the trachea and cricoid cartilage [3]. The thyroid gland is dissected from the trachea on both sides sectioning the isthmus. The second and third tracheal rings are punctured and the endotracheal tube is inserted at that point. Then the paratracheal areas are dissected. If necessary, a thyroid lobectomy or total thyroidectomy is performed. The posterior wall of the trachea is dissected and separated from the esophagus [6]. The hyoid bone is separated from the strap muscles. The thyroid cartilage is dissected from the surrounding tissue and thoroughly mobilized. This step is followed by a transoral robotic excision [4].

Retractors are used to retract and hold the laryngopharyngeal structures. The robotic system is docked and a 30-degree telescope is placed in the middle arm. The Schertel or Maryland forceps is placed onto the left robotic arm and the spatula monopolar cautery is fitted onto the right arm. The dissection begins by incising the anterior vallecula and pharyngoepiglottic fold. Then the hyoid bone is dissected from the preepiglottic area to reach the thyroid cartilage. The anterior pyriform sinus mucosa is incised and the superior laryngeal suspensory ligaments are dissected. Afterwards, the postcricoid area is preserved, starting from the stomach, and an upward dissection is performed. At this stage, direct visualization facilitates the identification of tumor margins, and the remaining tissue is dissected, and the larynx is removed transorally $[5,6]$.

The pharyngeal mucosa is sutured to the tongue base and the pharyngotomy is closed using 3-0 absorbable suture. When the neck is closed, superior skin retraction supports the pharyngotomy. The strap muscles are reapproximated over the pharyngotomy site. Suction drains are placed superior to the stoma, and the stomal suturing is completed to finish the procedure [3].

Postoperative complications are similar to open technique. Intraoperative complications have not been reported in the literature. The duration of the surgery has been estimated to be around $4 \mathrm{~h}$ and 30min [4-6]. Postoperative complications included esophageal fistula and hemorrhage [4,5]. Krishan et al [4] reported an average hospital stay of 21 days.

\section{The advantages of TORTL include}

1) Good cosmetic results, 2) Superior visualization with threedimensional imaging, 3) Suppression of physiological tremor, and 4)
The procedure allows surgical manipulation within a small working space.

\section{The disadvantages of TORTL include}

1) High cost, 2) Lack of tactile feedback, 3) Suitable only for selected patients, 4) The long operation time, and 5) Requires extensive experience.

Although TORTL is in its infancy, previous studies support its clinical applicability. The cosmetic benefits of this procedure are not as great as those associated with other robotic head and neck techniques because a permanent tracheostoma is created. However, as experience increases, the procedure may significantly minimize postoperative morbidities.

\section{References}

1. Dombrée M, Crott R, Lawson G, Janne P, Castiaux A, Krug B. Cost comparison of open approach, transoral laser microsurgery and transoral robotic surgery for partial and total laryngectomies. Eur Arch Otorhinolaryngol. 2014; 271: 2825-2834

2. Mendelsohn $\mathrm{AH}$, Remacle $\mathrm{M}$. Transoral robotic surgery for laryngeal cancer. Curr Opin Otolaryngol Head Neck Surg. 2015, 23: 148-152.

3. Lawson G, Mendelsohn AH, VanderVorst S, Bachy V, Remacle M. Transoral Robotic Surgery Total Laryngectomy. Laryngoscope. 2013; 123: 193-196.

4. Krishnan G, Krishnan S. Transoral Robotic Surgery Total Laryngectomy: Evaluation of Functional and Survival Outcomes in a Retrospective Case Series at a Single Institution.

5. Dowthwaite S, Nichols AC, Yoo J, Smith RV, Dhaliwal S, Basmaji J, et al. Transoral robotic total laryngectomy: Report of 3 cases. Head Neck 2013; 35: 338-342.

6. Smith RV, Schiff BA, Sarta C, Hans S, Brasnu D. Transoral robotic total laryngectomy. Laryngoscope. 2013; 123: 678-682.

7. Funk E, Goldenberg D, Goyal N. Demonstration of transoral robotic supraglottic laryngectomy and total laryngectomy in cadaveric specimens using the Medrobotics Flex System. Head Neck. 2017; 39: 1218-1225.

8. Schuler PJ, Hoffmann TK, Veit JA, Rotter N, Friedrich DT, Greve J, et al. Hybrid procedure for total laryngectomy with a flexible robot-assisted surgical system. Int J Med Robot. 2017;13.

9. Dionigi G, Lavazza M, Wu CW, Sun H, Liu X, Tufano RP, et al. Transoral thyroidectomy: why is it needed? Gland Surg. $2017 ; 6: 272-276$. 